\title{
THERMAL AGING AND CATALYST CONCENTRATION EFFECTS ON THERMO-DYNAMICAL AND MECHANICAL PROPERTIES OF A POLYESTER FIBERGLASS COMPOSITE
}

\author{
Salim Belaid $^{* 1}$, S.F. Chabira ${ }^{1}$, P. Balland ${ }^{2}$, A. Maati ${ }^{1}$, M. Sebaa $^{1}$ \\ ${ }^{1}$ Mechanics Laboratory, Department of Mechanics, Amar Telidji University, \\ Laghouat 03000, Algeria \\ ${ }^{2}$ SYMME Laboratory, Univ. Savoie Mont Blanc, FR-74000 Annecy, France
}

Received 31.03.2021

Accepted 07.06.2021

\begin{abstract}
This work deals with the characterization by physicochemical and mechanical analysis performed on composite polyester fiberglass plates molded by contact and aged in thermo-stated ovens to simulate their deleterious environment of use. The DSC analysis revealed that the catalyst concentration affected the thermal behavior of the composite. Indeed, the addition of a higher catalyst concentration slightly lowered the glass transition temperature. From a mechanical point of view, it was observed that the catalyst addition made the material stiffer. The properties at fracture were also affected by the catalyst concentration and varied irregularly with aging.
\end{abstract}

Keywords: composite; catalyst; endothermic peak; glass transition temperature; differential scanning calorimetry.

\section{Introduction}

Orthotropic fiberglass composite materials are very versatile and are utilized in a variety of applications. Because of their ease of molding, good aesthetic appearance, constant improvement of their properties, they occupy a prominent place in the plastic materials market. Composite parts provide superior strength, stiffness, and lightweight; therefore, they can be used for large complex-shaped structures, such as covers and tanks. They are also utilized where high-performance is required such as aerospace, race cars, boating, sporting goods, and industrial applications. The most widely used composite material is fiberglass in polyester resin, which is commonly referred to as fiberglass. Fiberglass is lightweight, corrosion resistant and economical, easily processed and has good mechanical properties. It is the dominant material in most industries such as boat

${ }^{*}$ Corresponding author: Belaid Salim, s.belaid@lagh-univ.dz 
building and corrosion protection equipment. It plays a major role in industries such as civil engineering, automotive, medical, recreational and industrial equipment.

However, as with most plastic materials, glass reinforced polyester is sensitive to environmental parameters such as sunlight, temperature, and various types of mechanical stresses. To anticipate or prevent an early failure of the material or even to estimate its service lifetime, aging studies can be regarded as a good alternative to understand how these different environmental parameters can interact deleteriously on the various properties of this material. Therefore, during an aging protocol, test pieces are picked up at regular intervals and different characterization analyses are carried out in order to check the progress of the material properties with aging.

Physical-mechanical characterization employing different techniques such as differential scanning calorimetry (DSC), differential thermal analysis (DTA), dynamic mechanical analysis (DMA), tensile tests, and microscopy can be regarded as very efficient techniques bringing useful information on the microstructural changes undergone by the composite consequently to aging, leading in a dramatic fall of the mechanical properties [1-6].

In the present work, orthotropic glass-reinforced polyester plates prepared by contact molding were subjected to thermal aging. Then physicochemical and mechanical characterizations were carried out on samples picked up at various stages of the aging protocols. This has shown how aging has adversely affected the mechanical properties such as longitudinal elastic modulus, stress and strain at fracture. The thermal analyses performed by DSC brought a good insight into the morphological changes undergone by the material thanks to the glass transition temperature (Tg), melting peak $(\Delta \mathrm{H}$ melting) and melting temperature (Tm) measurement.

Thermogravimetric analysis via the continuous measurement of the mass variation of a material sample over time allows evaluating its thermal stability.

Therefore, this work aimed to show how the longitudinal tensile Young's modulus, the strain, and stress at fracture change with exposure time. The thermal properties (such as the melting enthalpy, the glass transition temperature, the weight loss) were checked with exposure time. A second aspect, not the least, was to highlight the effect of the catalyst concentration used for the polyester resin curing on the properties just described above [7-9].

Finally, based on the different characterization results obtained it had become possible to highlight the relationship existing between the microstructural changes undergone by the material and its properties.

\section{Experiment}

\section{Polyester fiberglass composite preparation}

The bidirectional composites with crossed plies were prepared by contact molding. The volume fraction of fiberglass is $40 \%$ (E Roving SICOMIN BX 600 fiberglass, of woven Taffeta type, $600 \mathrm{~g} / \mathrm{m} 2$ ). The volume fraction of the polyester resin is then $60 \%$. It is marketed under the trade name, NORSODYNE® H 44281 TA. It is an unsaturated orthophthalic acid diluted with styrene moderately reactive, with a low viscosity. The 
catalyst used is a solution of peroxide PMEC 50 (i.e., $50 \%$ by weight solution of Methyl Ethyl Ketone Peroxide (MEKP) in a $9 \%$ to $9.2 \%$ titrating solution of active oxygen) [10]. The different constituents of the glass-fiber are reported in Table 1. Their respective concentrations are given in weight percent.

Before starting the molding operation, the surface of the mold was thoroughly cleaned (with acetone) to remove dust and dirt, if any. Then, the aluminum mold $(500 \mathrm{x}$ $500 \mathrm{~mm}^{2}$ ) was covered with a mold release agent (MOLD WIZ-F57 CN). The molding method consists in depositing sequentially in the mold a crossed ply of fiberglass (i.e., $0^{\circ}$ and $90^{\circ}$ fibers direction) on which a resin layer is deposed with a brush. To remove air bubbles that can affect the wetting of the fiberglass mat, a metal roll is passed with pressure on the surface. This operation was repeated six times (i.e., six plies) until the desired thickness was reached. Once dry, the obtained plate of $3.2 \mathrm{~mm}$ thickness is then gently removed from the mold. The aluminum mold, the taffeta, and the polyester fiberglass composite plate obtained after molding are represented in (Fig. 1).

Table 1. A mass ratio of the components of the E-glass fiber.

\begin{tabular}{lc}
\hline Components & mas.\% of glass E \\
\hline $\mathrm{SiO}_{2}$ & $53-55$ \\
$\mathrm{Al}_{2} \mathrm{O}_{3}$ & $14-15$ \\
$\mathrm{CaO}$ & $17-23$ \\
$\mathrm{MgO}$ & 1 \\
$\mathrm{Na}_{2} \mathrm{CO}_{3}$ & 0,8 \\
$\mathrm{~B}_{2} \mathrm{O}_{3}$ & $0-8$ \\
$\mathrm{Fe}_{2} \mathrm{O}_{3}$ & 0.3 \\
$\mathrm{TiO}_{2}$ & 0.5 \\
$\mathrm{ZrO}_{2}$ & $/$ \\
\hline
\end{tabular}
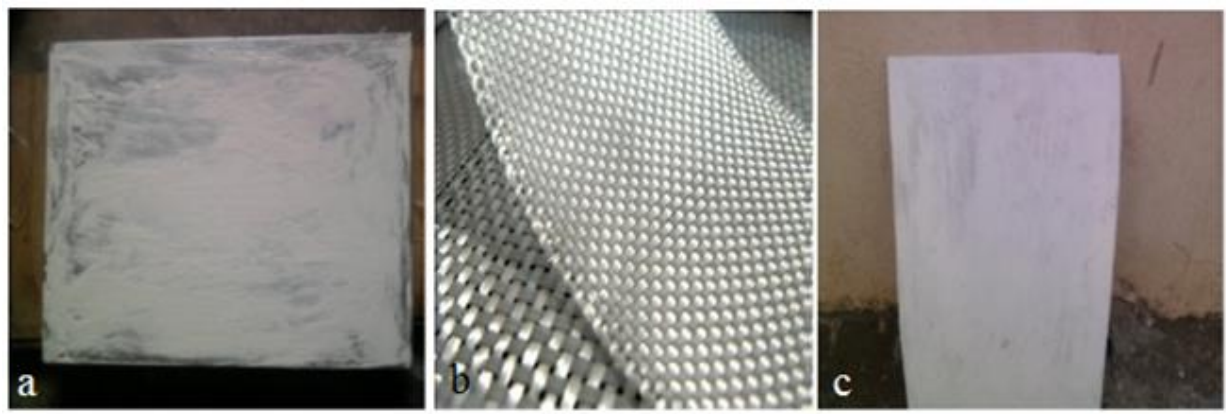

Fig. 1. Preparation of the polyester fiberglass composites: a) casting aluminum plate, $b$ ) fiberglass taffeta, c) Polyester fiberglass composite. 


\section{Thermal aging protocols}

After a sufficiently long post-hardening time, the plates were placed in ovens to undergo thermal aging at $80^{\circ} \mathrm{C}$ and $100^{\circ} \mathrm{C}$ respectively. The ovens (Memmert, UN 110, Germany) are thermo-regulated at $\pm 0.5^{\circ} \mathrm{C}$. The overall duration of the aging protocol is 150 days. Sampling was done every 30 days.

\section{Thermal analysis}

The measurement was carried out using a SETARAM TG/DSC model 92-1750 instrument. A small amount of material was encapsulated in an aluminum sample-pan. Then, the whole was heated from room temperature up to $800{ }^{\circ} \mathrm{C}$ in an inert atmosphere $\left(\mathrm{N}_{2}\right)$ with a heating rate of $10^{\circ} \mathrm{C} / \mathrm{min}$.

\section{Mechanical testing}

The mechanical characterization was carried out at room temperature $\left(25^{\circ} \mathrm{C}\right)$. The test was run after the samples were removed from the oven to ensure they had reached room temperature, but not too long after to avoid an uncontrolled structural change.

The tensile tests were performed on an INSTRON 5569 machine at fixed crosshead speed $(2 \mathrm{~mm} / \mathrm{min})$. The specimens were cut into a rectangular shape, according to the standard ISO574-4 for composite materials, with the following dimensions: width $=25$ $\mathrm{mm}$, length $=250 \mathrm{~mm}$ and thickness $=3.2 \mathrm{~mm}$. The stress-strain curve was the observable change in stress and strain of the composite sample obtained thanks to a $50 \mathrm{kN}$ load cell and a $12.5 \mathrm{~mm}$ extensometer.

\section{Results}

Thermal analysis - Catalyst concentration effect on the weight loss during the thermogravimetric test

Fig. 2 reports the weight loss variation as a function of temperature for three different kinds of material, virgin resin (i.e., without catalyst), resin with $2 \%$ catalyst and resin with $3 \%$ catalyst. For the virgin resin, the weight loss reached almost $92 \%$. Once compounded with the catalyst the weight loss decreased drastically to become $85 \%$ for $2 \%$ catalyst and $75 \%$ for $3 \%$ catalyst.

Fig. 3 is reported the variation of the weight loss as a function of the thermal aging time for the two different catalyst concentrations. It could be still noticed that with $2 \%$ catalyst (Fig. 3a) the weight loss tends to decrease with aging time while with $3 \%$ catalyst (Fig.3b) the weight loss varies irregularly but with a general trend of increasing with the increasing aging time. In Table 2 are reported the weight losses $\Delta \mathrm{m}_{\mathrm{i}}$ and their corresponding temperature $T_{i}$ determined at the inflection point of the $T G$ curves (Fig. 2). It appears that $\Delta \mathrm{m}_{\mathrm{i}}$ became slightly more important with the addition of the catalyst. Whereas, one would have expected that an increase in the concentration of the catalyst would have had the opposite effect. However, it can be still noticed that their corresponding temperatures regularly increase with the increasing catalyst concentration. This allows suggesting that the catalyst delays the composite decomposition rate. 


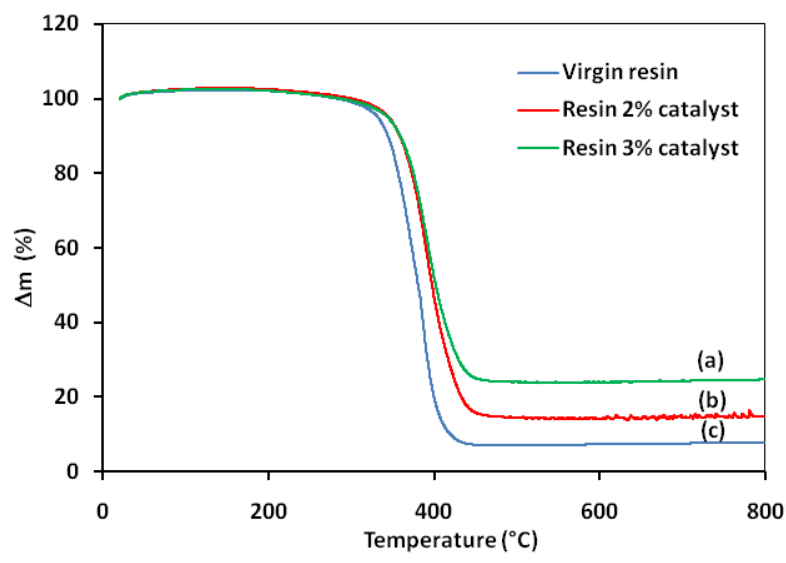

Fig. 2. Weight loss variation as a function of temperature for: (a) Virgin resin; (b) Resin with 2\% catalyst; (c) Resin with $3 \%$ catalyst.
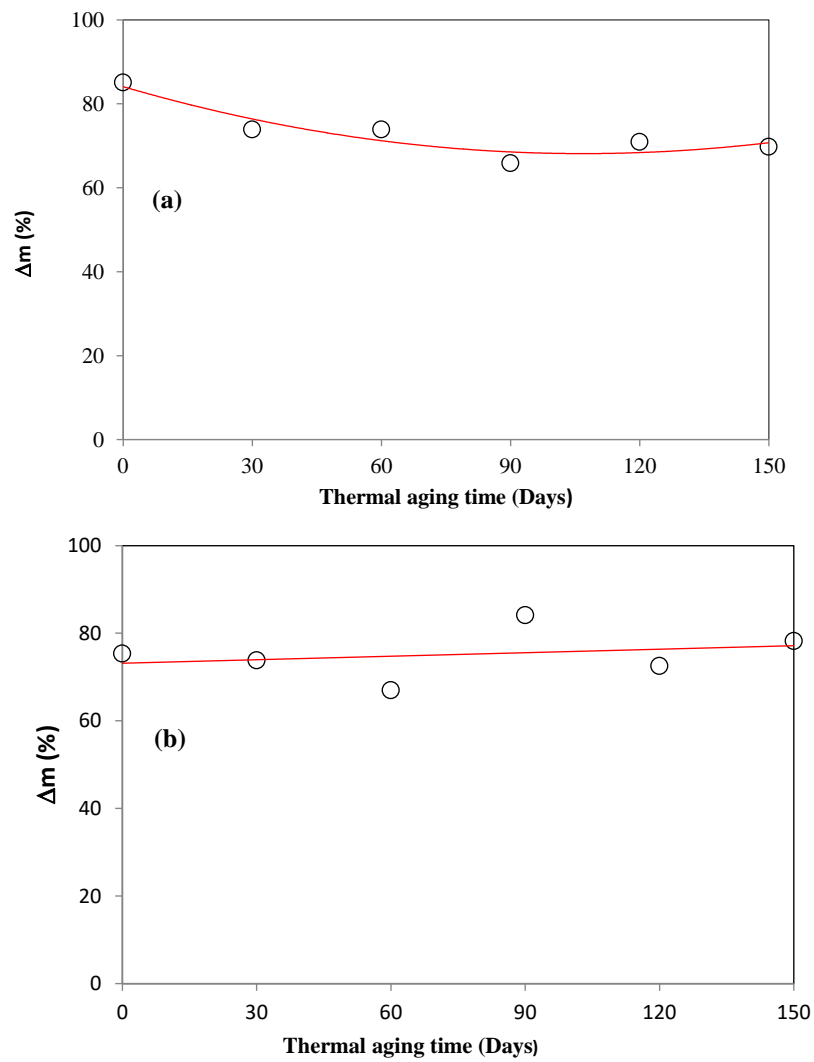

Fig. 3. Weight loss variation as a function of thermal aging time of the resin with (a) $2 \%$ catalyst, (b)3\% catalyst. 
Table 2. Weight loss and temperature at the inflection point for the virgin resin and those with $2 \%$ and $3 \%$ catalyst.

\begin{tabular}{lll}
\hline Material & $\Delta \mathrm{m}_{\mathrm{i}}(\%)$ & $\mathrm{T}_{\mathrm{i}}\left({ }^{\circ} \mathrm{C}\right)$ \\
\hline Virgin resin & 52 & 381 \\
$2 \%$ & 59 & 394 \\
$3 \%$ & 57 & 397 \\
\hline
\end{tabular}

\section{Catalyst concentration effect on the different thermal transitions}

In Figure 4 are reported the DSC curves of the resin with both $2 \%$ and $3 \%$ catalyst. On the DSC curve at temperatures ranging from 70 to $115^{\circ} \mathrm{C}$ appears a plateau; this corresponds to a phase transition. [11]. It corresponds very probably to the glass transition of the polyester resin. The increase of the catalyst concentration reduced the plateau width and height. This indicates that the transition started to appear slightly earlier for higher catalyst concentrations i.e., $76.7^{\circ} \mathrm{C}$ for $3 \%$ catalyst against $78.6^{\circ} \mathrm{C}$ for $2 \%$. The glass temperature $\mathrm{T}_{\mathrm{g}}$ determined at the inflection point of what looks like a plateau is $101.74^{\circ} \mathrm{C}$ for the composite with $3 \%$ catalyst and $103.73^{\circ} \mathrm{C}$ for the one with $2 \%$.

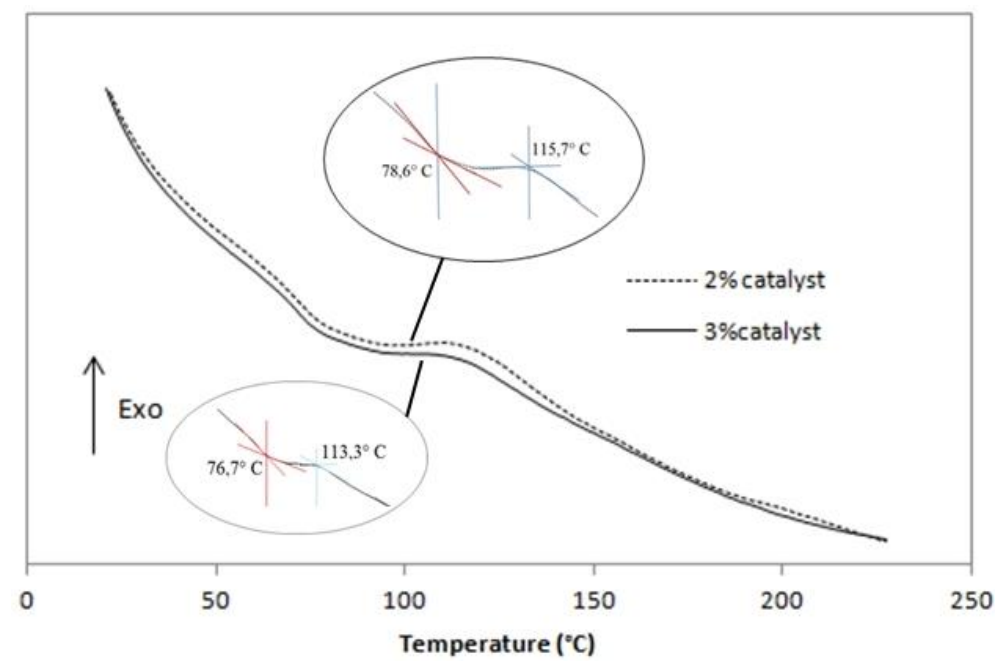

Fig. 4. Effect of the catalyst concentration on the aspect of the glass transition region in the DSC thermo-grams of the unaged resin with $2 \%$ and $3 \%$ catalyst.

\section{Catalyst concentration effect on the mechanical properties}

In figure 5 are reported the stress-strain curves of the two composites with 2 and $3 \%$ catalyst. It appears clearly that the catalyst concentration affects the mechanical behavior of the composite since with $3 \%$ catalyst it shows higher stresses during the tensile test compared to the one with $2 \%$. This allows suggesting that the catalyst concentration tends to strengthen more the resin during curing. This reveals that the number of cross-linking between the molecular chains is enhanced with the catalyst concentration. Even during aging, the catalyst concentration has a good effect on the mechanical properties of the composite and this is described in more detail in the coming section. 


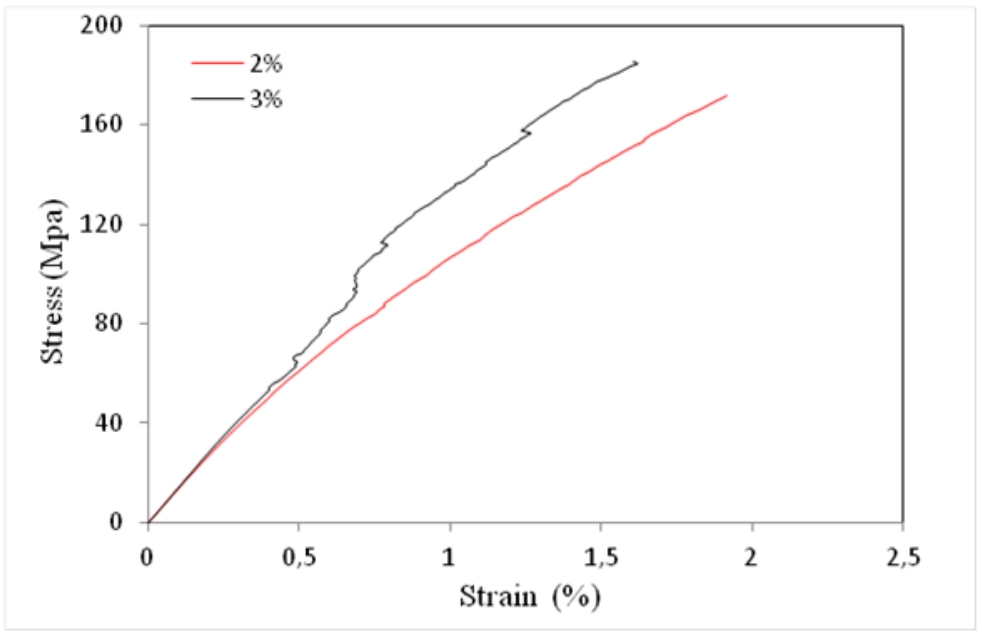

Fig. 5. Catalyst concentration effect on the stress curve of the polyester fiberglass composite.

\section{Discussions of the aging effect on}

\section{Glass transition}

In Figure 6 are reported the DSC curves of the composite with 2\% catalyst and corresponding to the temperatures ranging from room temperature up to $230^{\circ} \mathrm{C}$. As described just above the glass transition appears as a plateau for the unexposed material. With aging time the aspect of the curves changes and the baseline goes down and up irregularly.

With $3 \%$ catalyst, it appears a batch of curves (Fig. 7). They vary irregularly up or down but they remain closer to each other compared to those of the composite with $2 \%$ catalyst.

Table 3. The glass transition temperature of the composite with $2 \%$ and $3 \%$ catalyst at different aging stages with a 30-day progression step.

\begin{tabular}{llllllll}
\hline \multicolumn{2}{l}{ Aging time (days) } & 0 & 30 & 60 & 90 & 120 & 150 \\
\hline \multirow{2}{*}{$\operatorname{Tg}\left({ }^{\circ} \mathrm{C}\right)$} & $2 \%$ catalyst & 103.7 & 117.5 & 115.4 & 125 & 132.1 & 131.1 \\
\cline { 2 - 8 } & $3 \%$ catalyst & 101.7 & 129.4 & 124 & 121.5 & 117.5 & 131.8 \\
\hline
\end{tabular}

In both cases, the plateau appearing on the curves of the two unaged composites immediately disappeared once exposed. The glass transition temperature determined on the different curves is reported in Table 3. It globally increased with exposure time. For the composite with $2 \%$ catalyst, it increased by about $26 \%$, while for that with $3 \%$ catalyst is increased by $31 \%$. 


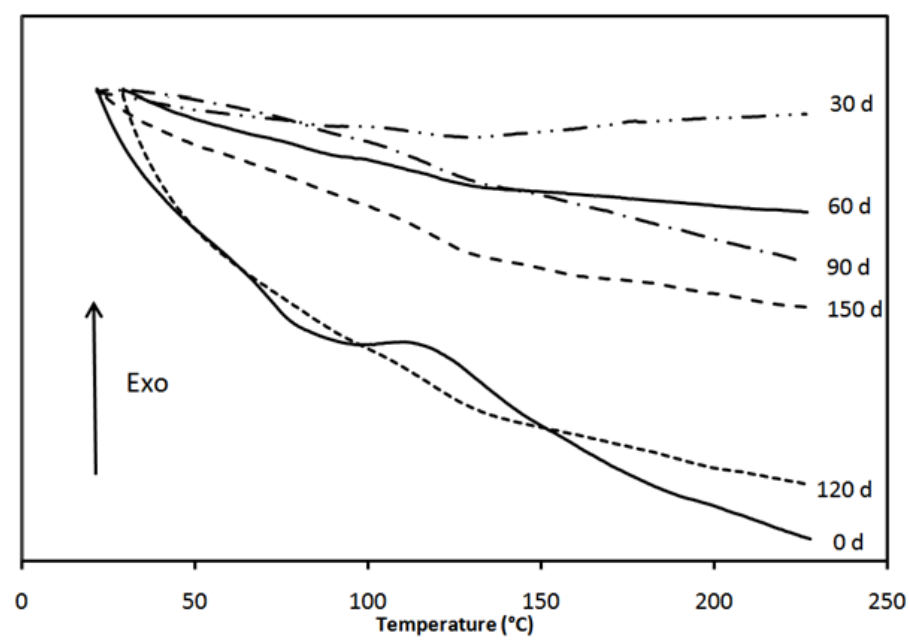

Fig. 6. Aging effects on the glass transition region aspect of the DSC thermograms of the composite with $2 \%$ catalyst.

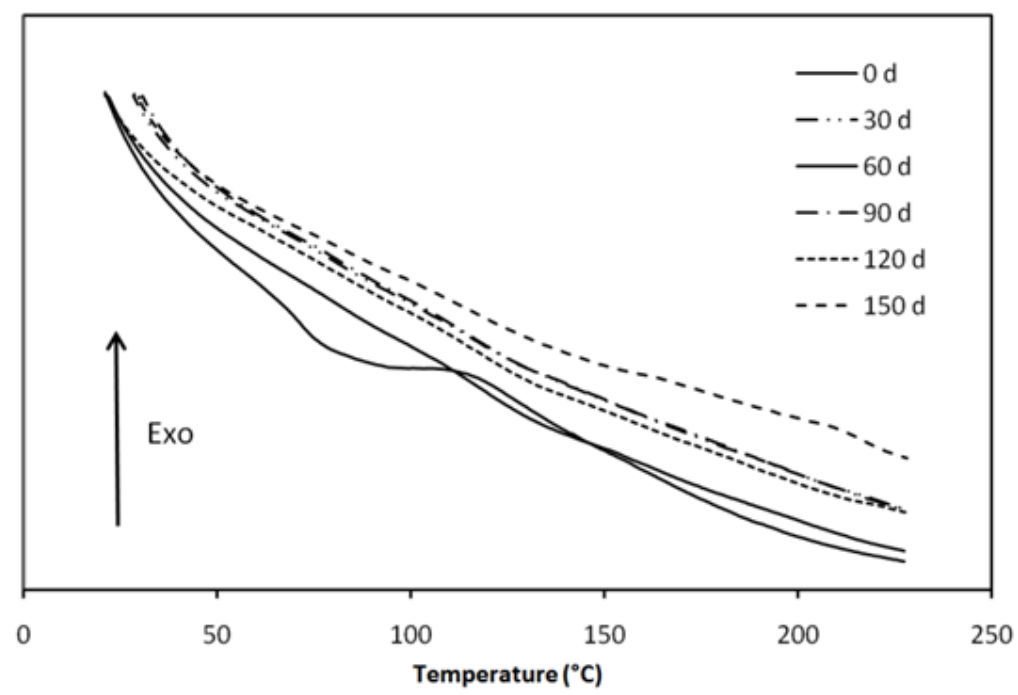

Fig. 7. Aging effects on the glass transition region aspect of the DSC thermo-grams of the composite with $3 \%$ catalyst.

The disappearance of the plateau in both cases reveals that the polyester resin is the subject of major molecular changes such as chain scissions very frequent in polymeric materials when subjected to thermal treatment at relatively elevated temperature. As a matter of fact, in the following section, the other thermal parameters are also very affected by this treatment supporting as such our hypothesis. 


\section{Melting}

In figures 8 and 9 are reported respectively the melting peaks of the composite with $2 \%$ and $3 \%$ catalyst [12]. The peak temperatures at the maximum of the broad endotherms are reported in Table 4.

It can be observed that the melting temperature is the same for the unexposed samples and varies irregularly with increasing aging time. In both cases, the minimum Tm was reached after 30 days exposure and then increased again. The end value of Tm is higher for the composite with $3 \%$ catalyst than for the one with $2 \%$. In the same vein, the melting enthalpies of the two composites are reported in Table 5.

The enthalpy variation decreases irregular but reveals a downward trend more pronounced for the composite with $3 \%$ catalyst.

Table 4. Melting temperature of the two composites with respectively 2 and 3\% catalyst.

\begin{tabular}{llllllll}
\hline Aging time (days) & 0 & 30 & 60 & 90 & 120 & 150 \\
\hline \multirow{2}{*}{$\begin{array}{l}2 \% \text { catalyst } \\
\text { Tg }\left({ }^{\circ} \mathrm{C}\right)\end{array}$} & 390 & 370 & 371.4 & 376.4 & 378.6 & 378.6 \\
& $3 \%$ catalyst & 390 & 376.4 & 380.7 & 381.4 & 387.9 & 387.1 \\
\hline
\end{tabular}

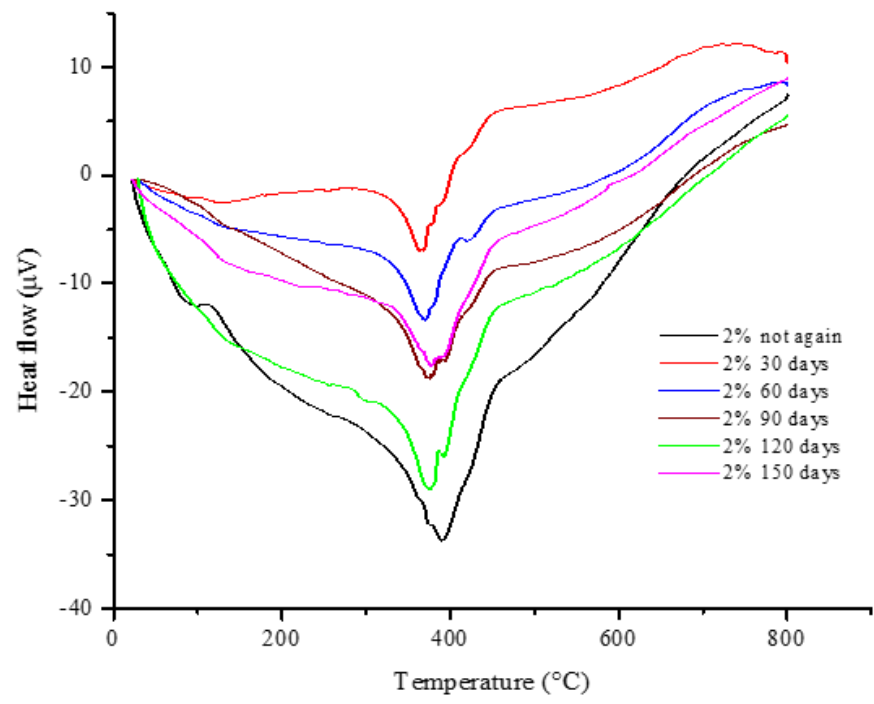

Fig. 8. Aging effect on the melting peak aspect of the composite with $2 \%$ catalyst. 


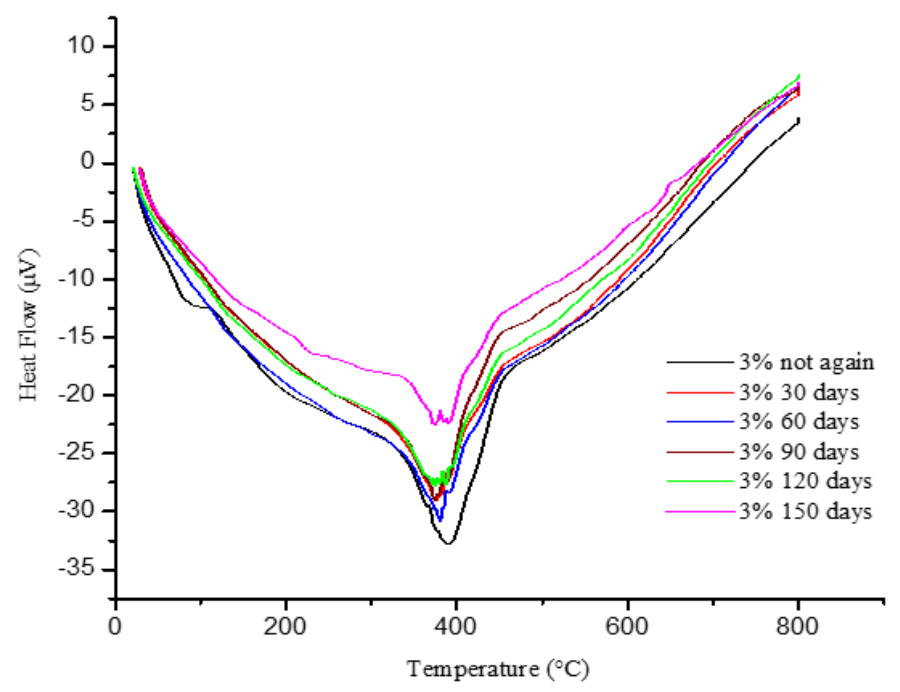

Fig. 9. Aging effect on the melting peak aspect of the composite with $3 \%$ catalyst.

Table 5. Melting enthalpy of the two composites.

\begin{tabular}{lll}
\hline $\begin{array}{l}\text { Aging time } \\
\text { (days) }\end{array}$ & $\begin{array}{l}\text { Enthalpy (endothermic pic) }(\mu \mathrm{V} \cdot \mathrm{smg}) \\
2 \% \text { catalyst }\end{array}$ & $\begin{array}{l}\text { Enthalpy (endothermic pic) } \\
(\mu \mathrm{V} \cdot \mathrm{smg}) \\
3 \% \text { catalyst }\end{array}$ \\
\hline 0 & 190 & 225.09 \\
30 & 70 & 208.94 \\
60 & 77 & 186.29 \\
90 & 131 & 71.71 \\
120 & 141 & 68.32 \\
150 & 169 & 10.85 \\
\hline
\end{tabular}

\section{Mechanical properties}

In fig. 10 and 11 are reported the stress-strain curves of the two composites with 2 and $3 \%$ catalyst. It appears clearly that aging affects the mechanical behavior.

A cursory observation of the two figures reveals that the catalyst concentration affected the global behavior of the composite. For the composite with $2 \%$ catalyst, it appears a batch of curves quite close to each other. This seems to indicate that aging did not affect noticeable the global stress-strain behavior of the composite. While for the one with $3 \%$ catalyst the smoothness of the curves is more affected, especially for the first and last aging time. The curves are less close to each other.

For better showing the effect of aging on the evolution of the Young's modulus, maximum stress and strain, determined from the previous figures, are respectively reported as a function of aging time in figures 12 and 13.

The Young's modulus $\left(\mathrm{E}_{\mathrm{L}}\right)$ is measured during the tensile test in the longitudinal direction. It is determined from the slope of the linear part of the stress-strain curve. The 
variation of the Young's modulus $\mathrm{E}_{\mathrm{L}}$ as a function of the aging time for the composite with 2 and $3 \%$ by weight catalyst respectively (Fig $12 \mathrm{a}$ and b) shows that it increases very slightly with exposure time but globally varies irregularly. The measurement of the modulus before aging shown that, the modulus is a little bit higher for the composite with $3 \%$ catalyst. Even, during aging the catalyst maintained this tendency since the average value for the whole duration of exposure is $13.5 \mathrm{GPa}$ for the composite with $2 \%$ catalyst against $14.1 \mathrm{GPa}$ for the one with $3 \%$. It can be observed that in both cases the modulus is the lowest after 30 days exposure, as already seen for the melting temperature. This supports the previous assumption that major structural changes took place at this period of the thermal aging.

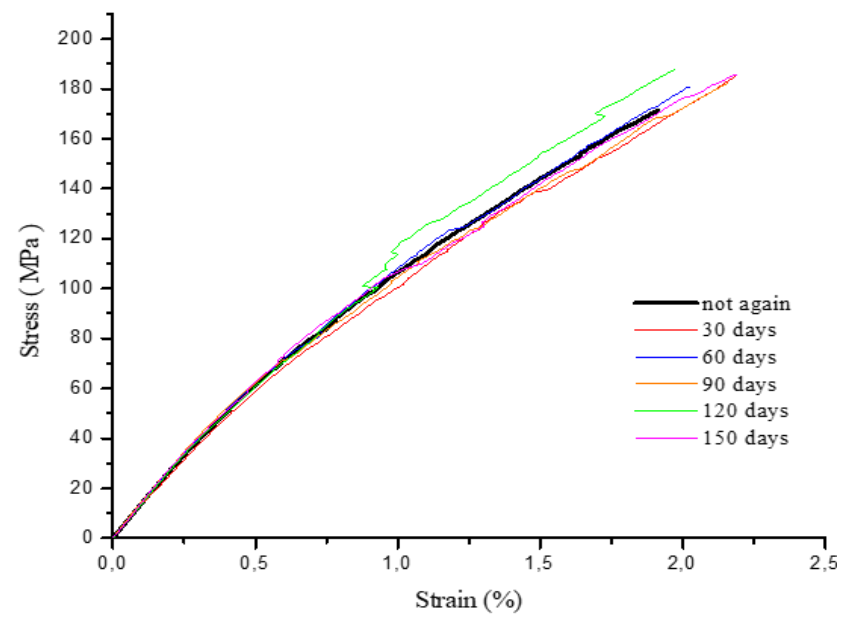

Fig. 10. Effect of aging on the stretching behavior of the composite with $2 \%$ catalyst.

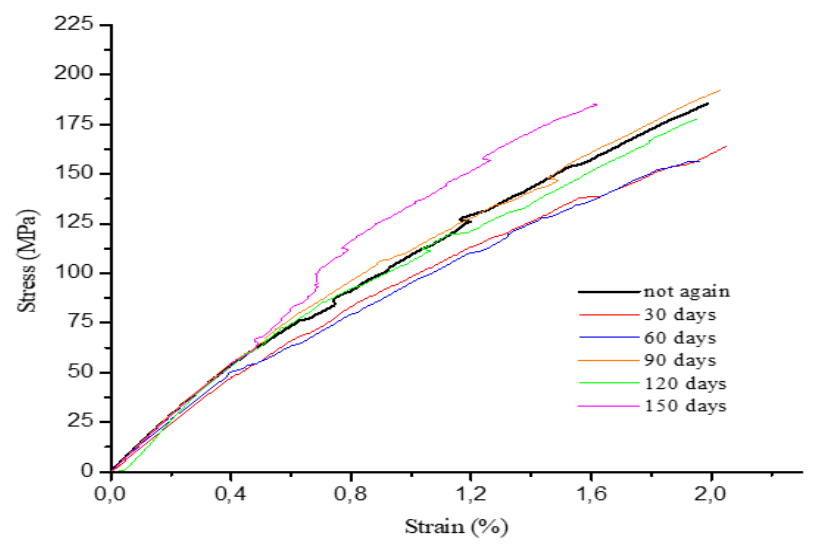

Fig. 11. Effect of aging on the stretching behavior of the composite with 3\% catalyst. 

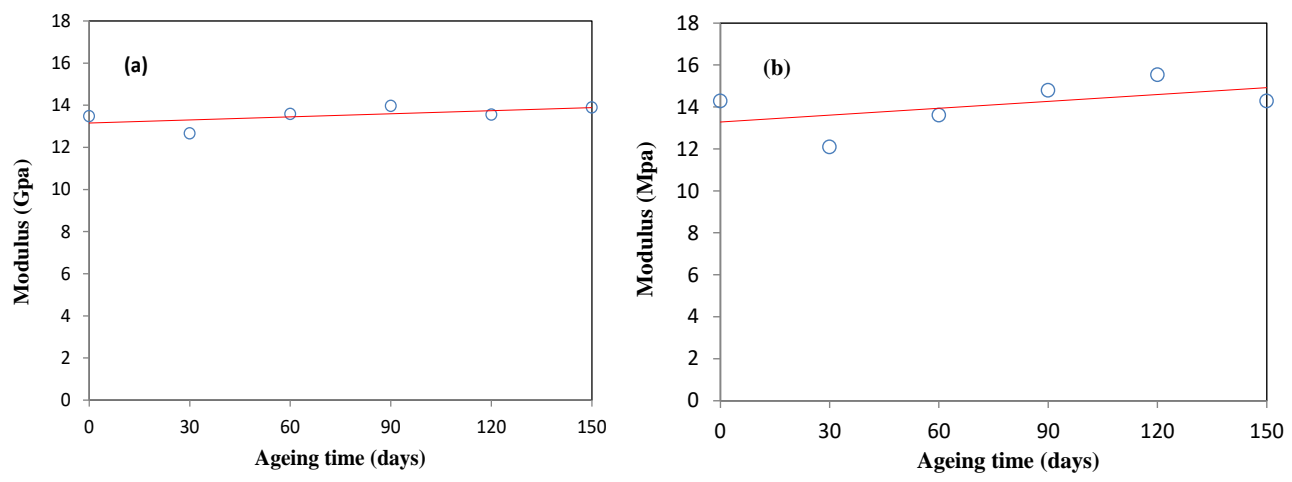

Fig. 12. Effect of aging on the Young's

modulus for the composite (a) with $2 \%$ catalyst, (b) with $3 \%$ catalyst.

The representation of stress and strain at fracture (the last point of the tensile curve) versus exposure time in Figure 13 reveals, as observed for the Young's modulus an irregular variation.

For the unexposed composites a higher catalyst concentration decreased the strain at fracture, which is indeed lower of about $19 \%$ for the composite with $3 \%$ catalyst. The opposite effect is observable for the stress at fracture since the stress at fracture for the composite with $3 \%$ catalyst is greater from about $8 \%$. These results reinforce the previous assumption indicating that the catalyst concentration tends to increase the rate of cross linking. Indeed, it is well known that cross linking decreases the molecular chains mobility meaning that they cannot flow past one another upon the deformation of the bulk. Therefore, the chains reach their mobility limit for smaller deformations, and the applied stress must be greater.

With aging time, stress and strain at fracture of the composite with $2 \%$ catalyst increased slightly but irregularly with exposure time. For the composite with $3 \%$ catalyst the stress and strain at fracture varied also very irregularly. It can also be noticed that stress at fracture seems to very slightly increase with exposure time while strain at fracture varies so irregularly that it remains difficult to find a general trend [13]. The variation of this mechanical greatness is the consequences of micro structural and morphological changes undergone by the matrix during aging and also very probably because of interfacial decohesion between the fiber and the resin. $[14,15]$. 

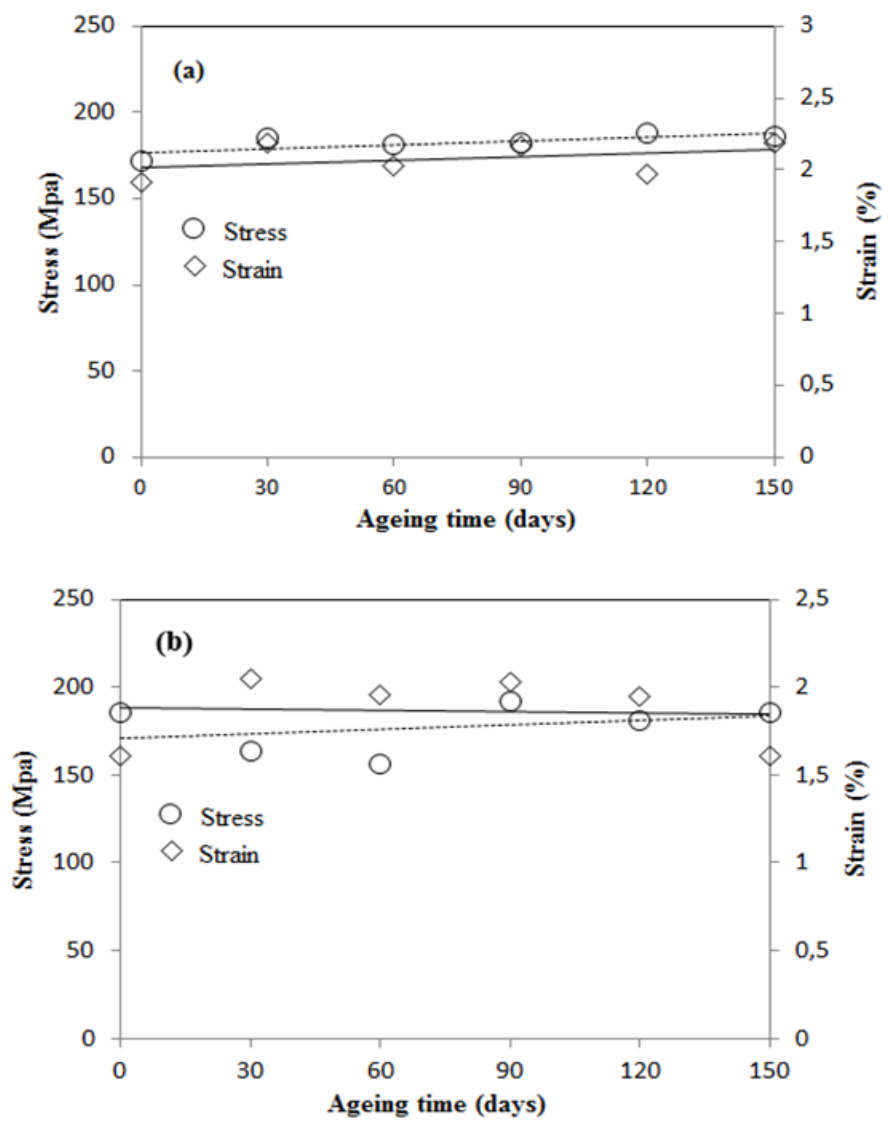

Fig. 13. Effect of aging on the stress and strain at break for the composite (a) with $2 \%$ catalyst (b) with $3 \%$ catalyst.

The images obtained by scanning electron microscopy (Fig. 14), for the composite with $3 \%$ catalyst aged at $100{ }^{\circ} \mathrm{C}$, seems to confirm the decohesion at the fiber/resin interface of this sample, where it can be see a clear degradation of the fibers and the matrix.
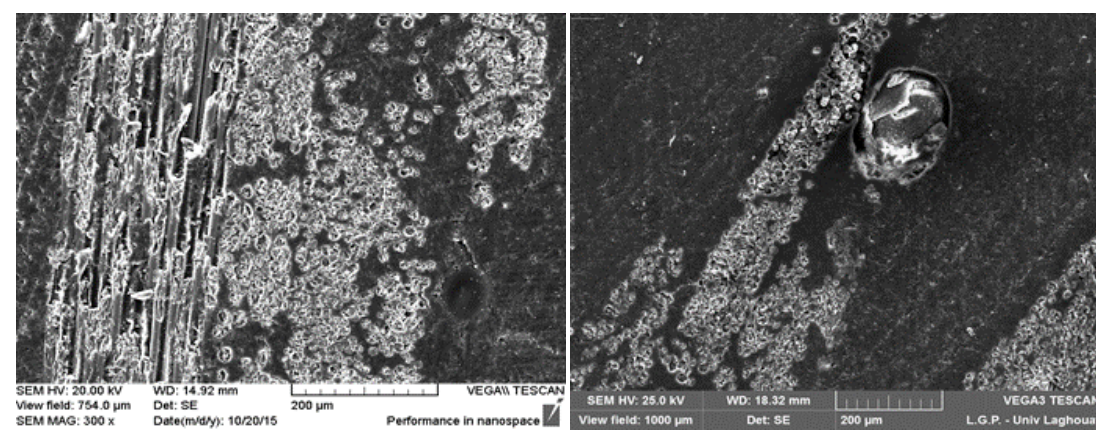

Fig. 14. SEM image of samples aged for 120 days at $100^{\circ} \mathrm{C}$ of the $3 \%$ catalyst composite. 
In the matrix, it appears cracks due to the change of the chemical structure where chain scissions must have played a key role. The holes observable in the pictures are also due to the removal of some matter very probably because of the removal of molecules from the matrix which are released in the state of gases. This explains the strong smell in the laboratory during the whole duration of the thermal treatment. Several mechanisms of thermal degradation of the polyester resin have been proposed in the literature. The mechanism represented by Anderson and Freeman [16] is illustrated in Fig. 15; this reaction is considered to be predominant during thermo-oxidative degradation. Oxygen attacks the styryl group and forms the hydroperoxide group.

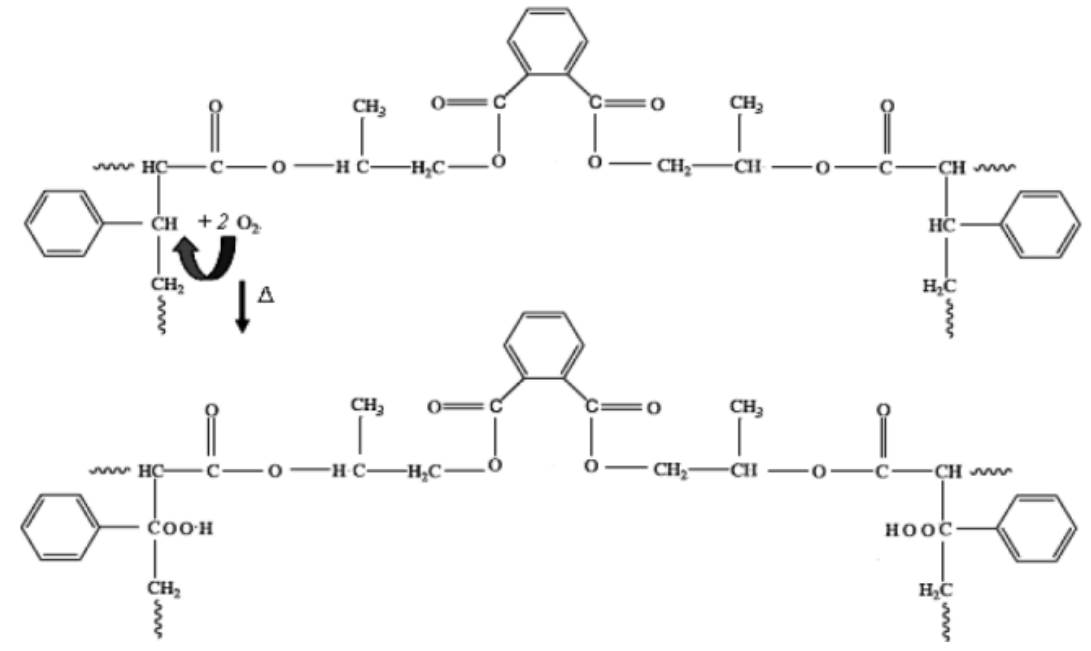

Fig. 15. Mechanism of formation of hydroperoxide groups during thermal degradation of the resin.

\section{Conclusion}

This study, performed on a composite material made of polyester resin reinforced with fiberglass, allowed to connect the evolution of the thermal properties with the mechanical ones besides the combined effects of thermal aging and catalyst concentration.

The thermo-gravimetric analysis showed that the catalyst delays the composite decomposition rate and lowers the glass transition temperature of the resin. However, with aging, the glass temperature $\mathrm{T}_{\mathrm{g}}$ increases in approximately the same proportion whatever the catalyst concentration. As for the melting temperature, it tended rather decrease with aging time, with a significant drop after 30 days of exposure. Indeed, the variations of the mechanical greatnesses such as the modulus, the properties at fracture and those measured by the thermal analyses strengthen this assumption that a major structural change takes place at this peculiar period of the aging protocol.

The polymeric matrix is the subject of an important competition between crosslinking and chain scission reactions affecting the microstructure of the material. This phenomenon explains the irregular variation of the mechanical and thermal properties of the material, where chain scission reactions lower the properties at fracture while cross 
linking increases them. It appears through this modest contribution that characterization techniques such as FTIR and DRX can be regarded as interesting means for future works in order to bring additional information on the microstructural changes undergone by the material during aging and to understand how these last ones affect the morphological and mechanical properties.

\section{References}

[1] A. Landesmanna, C.A. Serutia, E. deMiranda Batista: Materials Research, 18 (2015) 1372-1383.

[2] N. Delahaye, S. Marais, J. M. Saiter, M. Metayer: Journal of Applied Polymer Science, 67 (1998) 695-703.

[3] S.O. Adeosun, E.I. Akpan, H.A. Akanegbu: International Journal of Composite Materials, 5 (2015) 52-64.

[4] R. Alabbas, W. Khalil, S. Alhariry: Chemistry and Materials Research, 9 (2004) $15-20$.

[5] S. Belaid, S.F. Chabira, P. Balland, M. Sebaa, S. Belhouideg: J Mater Environ Sci, 6 (2015) 2795-2803.

[6] A. Christiansen, J. B. Shortall : Journal of Materials Science, 11 (1976) 11131124.

[7] H. Duan, L. Zhang, J. Wang, X. Yang: Journal of Wuhan University of Technology-Mater Sci. Ed. Aug (2008) 460-462.

[8] A. Hodzic, J.K. Kim, A.E. Lowe, Z.H. Stachurski: Composites Science and Technology, 64 (2004) 2185-2195.

[9] K. de la Caba, P. Guerrero, A. Eceiza, I. Mondragon: Polymer, 3 (1996) 275-280.

[10] J.L. Martin: Polymer, 40 (1999) 3451-3462.

[11] A. Khalyavina, L. Häubler, A. Lederer: Polymer, 53 (2012) 1049-1053.

[12] R. M. Schulken, R.E. Boy, R. H. Cox: Journal of polymer science Part C, 6 (1964) 17-25.

[13] Y.H.A. Mugahed, R. Alyousef, R.S.M. Rashid, H. Alabduljabbar, C.C. Hung: Structures, 16 (2018) 208-238.

[14] K. Johnand, S.V. Naidu: Journal of Reinforced Plastics and Composites, 23 (17) (2004) 1815-1819.

[15] G. Coulon, J. M. Lefebvre, B. Escaig: Journal Polymer, 12 (1984) 399-405.

[16] D.A Anderson, E.S Freeman: Journal Applied Polymere Science, 1 (1959) 192199.

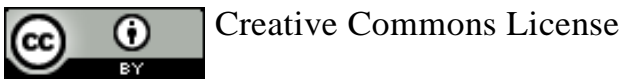

This work is licensed under a Creative Commons Attribution 4.0 International License. 